\title{
Artificial Intelligence: A Technological Prototype in Recruitment
}

\author{
R. Vedapradha, Ravi Hariharan ${ }^{(0)}$, Rajan Shivakami \\ Department of Commerce and Management, St. Joseph's College of Commerce (Autonomous), Bangalore, India \\ Email:vedahariharan@gmail.com,Hari712@gmail.com,shivakami.rajan@gmail.com
}

How to cite this paper: Vedapradha, R., Hariharan, R. and Shivakami, R. (2019) Artificial Intelligence: A Technological Prototype in Recruitment. Journal of Service Science and Management, 12, 382-390. https://doi.org/10.4236/jssm.2019.123026

Received: March 4, 2019

Accepted: April 12, 2019

Published: April 15, 2019

Copyright $\odot 2019$ by author(s) and Scientific Research Publishing Inc. This work is licensed under the Creative Commons Attribution International License (CC BY 4.0).

http://creativecommons.org/licenses/by/4.0/

(c) (i) Open Access

\begin{abstract}
Purpose: The study is conducted to evaluate the adaptability of artificial intelligence in recruitment and to assess the effect of this technology on the performance of the employees. Design/Methodology/Approach: Standard Multiple Linear regression model is used to predict the performance of the employees and one-way ANOVA is used to compare the artificial intelligence based recruitment with performance indicating variables namely reliability, productivity, Automation, Gamification \& Training using SPSS. Snowball sampling method has been adopted for a sample size of 440 respondents working in leading recruitment consultancies in urban Bangalore. Findings: There is a greater association between the recruitment and performance variables when artificial intelligence is adopted as it is significant at 0.001 per cent level and productivity being the maximum. However, the impact of implementing gamification for recruitment doesn't have a significant impact on the output due to partial significant effect on the adoption as $(\mathrm{p}=0.046<$ 0.05 ). Value of " $R$ " is 0.604 and the coefficient of determination is 0.365 . Productivity, Training, Automation \& Reliability are the significant predictors of the performance in employees. Originality/Value: Artificial intelligence has emerged as a boon to the recruiters by automating the repetitive tasks, administrative tasks. Intelligent screening helps in automating resume screening, recruiter Chatbots for real-time candidate engagement, and digitization of interviews. This promotes pro-active strategic decision making better by the recruiters.
\end{abstract}

\section{Keywords}

Artificial Intelligence (AI), Gamification, Recruitment and Performance

\section{Introduction}

Artificial Intelligence is an innovative technology emerging trend that can be 
witnessed in Human Resource technology, especially can be very effective and beneficial in the area of recruitment. It facilitates in reducing or eradicating the time-consuming activities, streamlining and automating the screening of resumes, matching the job requirements and available skills of the candidates more efficiently and effectively which enables in timely decision making by the professionals. It improves quality by recruiting standardized job matching qualities. Intelligent screening that automates the resumes, ensures a great avenue for the recruiters preventing disruption in the workflow, candidate workflow, and requires minimal information technology support. Volvo, leading Swedish Luxury Vehicles Company, converted their Brussels Motor Show into Job Fair at Belgium by creating an outstanding mark for themselves through their "Recruiting Car". The candidates were interviewed by the AI embedded car for the post of service technicians at the Volvo plant, who had to go through the process of recruitment by asking questions related to the concepts, requesting them to identify the parts through simulation.

\subsection{Role of Artificial Intelligence}

The innovative technology facilitates machines to learn ability to make decisions, logical thinking and react systematically to reap the benefits of deploying AI-based software in the recruitment transforming the majority of the process into automation that can be performed by a machine empowering with the ability to analyze big data faster and predict the probable outcomes. Currently, the solutions to the time-consuming recruitment have opened up through data-driven recruitment, improved candidate experience, social media recruiting, video job interview, AI-based screening of application forms. Virtual assistants are competent enough in connecting with candidates, storing, evaluating the applications and interact to assist recruiters in managing the database of the candidates for further reference. It is capable of scrutinizing the browsing history of the users in posting advertisements regarding the vacancies across companies for various jobs that are posted to the potential right candidates on the website pages. Chatbots are popularly used to extract basic information from the resumes of the candidates, which automatically matches the requirements with skill sets of the candidates. These are a blend of AI and natural language learning processing technology for better interaction like humans responding logically. Mya \& Olivia are the examples of Chatbots, engaged on the company's official website to filter the first level of screening when candidates visit their websites and also help them in completing the application process, schedule management, sending periodical updates to the candidates on application status, feedback system, instant response to the candidates on the basic queries. They also encourage in proactive bonding between company and candidates reducing the hiring cycle, creating a single source of reliability with passive candidates making them feel like customers. Most of the hiring managers are relying on Applicant Tracking System (ATS) software to improve the quality of hiring, automate the process of search- 
ing the prospective candidate matching the parameters picking up profiles from various sources like social media, job portals, networking sites to enhance the efficiency in the process, documents the employee records, maintain the compliance as per the government's regulatory framework [1].

\subsection{Current Application of Artificial Intelligence}

The acceptance of this innovative technology has been spreading its magic across many industries. Startup companies seem to be a pioneer in exploiting the merits of this technology to create an avenue to their business. Some of the companies already implemented AI in the recruitment process have been discussed below.

Skillate: SAP Labs incubation has AI platform enables them in scanning the resumes of the candidates from the database through social media to validate their bio-data to match their skillsets with job positions.

Hirevue: US-based Company applies video intelligence to analyze video interviews of candidates and give employers more insights to recruiters facilitating faster decisions.

Interviewed: San Francisco based company series of automated screening tools for programming tests, personality assessments, language skills where they undergo simulations before selection.

Entelo: It browses social profiles using predictive algorithms to determine the most probable candidate to leave their current job.

Koru: Automated online talent screening uses predictive analytics and AI to improve hiring outcomes in Investment banks.

Zoom \& Montage: These are teleconferencing AI-powered software to ease the recruiters to choose the suitable candidate through video conferencing for their process where there is provision for screening candidates for face to face across various rounds of interviews for the better understanding of the candidates.

Textio: It is based on the predictive data analysis with respect to the potential candidates applying to the high profile jobs in which the software analysis the meaningful and common language style of patterns from the documents posted marching towards the accuracy of the predictions based on the advertisements posted by the companies.

Panna: It is an AI hiring repository of dynamic questions, expert evaluation, documenting the interviewing, video conferencing, voice and face recognition which is designed on the machine learning to validate the applicants' video interviews to detect any malpractice or strange movements, investigating if someone is helping the candidate through other electronic devices offering intuitive interface.

Harver: Algorithms calculate the score of the candidate with respect to job profiles in line with the company's requirements facilitating to predict the chances of selected candidate succeeding the role after being recruited based on 
which the company can make wise decisions before hiring [2].

\section{Literature Review}

The authors proposed a constructive solution to the online recruiters to ensure not to omit the suitable candidate and to overcome tedious search techniques by blending implicit profiling method as well as automated collaborative filtering to benefit Job finder domains [3]. A Multiagent application has been adapted to recruitment and assesses the salesperson integrated with expert systems and soft computing techniques enabling to forecast their behavior towards the sales [4]. The recruitment process of matching the potential candidates with suitable job skills through conventional methods over online platforms causes damage to the company in terms of time, cost and resources [5]. Probabilistic automated recommendation approach using algorithm has been applied by the researcher to match the requirements of the company and skillsets of the candidates. An approach to predict the levels of the recruitment based on generic algorithms in Fisheries with an implementation of artificial intelligence related to spawning biomass resulting to be 61.9 per cent predicting correctly at 5 per cent mutation rate. It revealed that the spawning biomass wasn't significant for the establishment of recruitment level rather was based on the environment [6]. The emphasis of the study is on the recruitment theory focused on the communicative and cognitive economy built on the neural mechanisms with different strategies to address the challenges of communication during the process of recruitment [7]. Automation of the recruitment process has been consistent when tested for the applicants 'profile, semantically when compared with job requirements based on the linguistic analysis proving better than human recruiters in evaluating the applicants through online recruitment using machine learning algorithms [8]. The artificial intelligence facilitates in the recruitment process of an organization through a modern approach based on the logical reasoning, probability, mathematics, imperfect real-time decisions, game theories can be applied in the recruitment for better performance and to cut down the cost [9].

\section{Statement of the Problem}

Attrition has always been a pain point to most the organizations which causing a huge loss in terms of cost, resources, quality employees and taking a setback in knowledge sharing or downloading to the new employees. Hence the need of the hour is emphasizing the reduction in the cost of recruitment and increasing productivity with better quality. This can be achieved by implementing artificial intelligence in human resource recruitment.

\subsection{Objectives}

$\checkmark$ Evaluation of the adaptability of artificial intelligence in the recruitment of candidates by Human resource personnel in an organization.

$\checkmark$ Analysis of performance of employees with an implementation of artificial 
intelligence.

\subsection{Hypothesis}

$\mathrm{H}_{0}$ : There is no association between the recruitment and performance variables when artificial intelligence is adopted.

$\mathrm{H}_{0}$ : There is no significant influence of artificial intelligence on the performance of employees.

\subsection{Sampling}

Snowball sampling method is based on reference received from the known potential HR personnel respondent working in some of the leading consultancy firms forwarded the study questionnaire to their colleagues and friends of same organizations as well as other firms to help us complete the study. This method has been adopted to arrive at the sample size of 440 based on the references received from the friends working across the recruitment consultancies in urban Bangalore.

\subsection{Data \& Source}

The research is exclusively based on the primary data collected through a structured questionnaire from the respondents, Human resource employees working in leading recruitment consultancies in urban Bangalore.

\subsection{Variables \& Statistical Tools Used}

The variables chosen for the study consists of Quality, Automation, Decisions, Cost, Optimization, Accuracy, Payroll, Compliance, Data, Consistency, Paperless, Efficiency, Progress, Training, Branding, and Talent pool clustered into Productivity, Training, Automation, Reliability, and Gamification. Statistical Package for Social Science (SPSS) has been used to test Multiple Linear Regression Analysis and One-way ANOVA.

\subsection{Limitations}

The study has been confined only to consultancy firms. There is further scope for venturing into other firms and also can conduct comparative study between gamification \& artificial intelligence for recruitment. This also requires a lot of data and can learn human bias. Often the recruiters are skeptical about adopting the new technology due to automation doing away with their jobs.

\subsection{Scope for Further Research}

Artificial Intelligence is emerging trend that can be tapped to fit into various business process models to reap the benefits of accuracy, cost \& time effective and improved efficiency with great reliability. The researchers can conduct experiments of pre and post comparative study of implementation of $\mathrm{AI}$ in other industries, develop models to transform the business, explore the feasibility of 
application in the service industries like banks, e-commerce, hospitality, Investment Banks etc.

Table 1 reveals that there is a greater level of association between the AI enabled recruitment and the performance of the employees. The variables indicating the performance based on the reliability $(\mathrm{F}=12.426)$, Productivity $(\mathrm{F}=$ 43.625), Automation ( $\mathrm{F}=28.695)$, Training $(\mathrm{F}=36.798)$, and Gamification $(\mathrm{F}=$ 7.097) are highly statistically significant as the probability value is less than 0.001 .

Table 2 shows that there is a significant impact of artificial enabled recruitment which can be helpful in predicting the performance of the employees based on the reliability, productivity, gamification, automation, and training. The results obtained from the stepwise multiple regression analysis reflects training has the highest beta value (0.388), productivity $(0.287)$, and automation $(-0.306)$ significant at 0.01 per cent level but reliability $(0.159)$ and gamification $(-0.107)$ which is not significant as the " $\mathrm{p}$ " $>0.05$. The Unstandardized coefficients of productivity is $(0.084)$, training $(0.116)$, automation $(-0.065)$, reliability $(0.045)$ and gamification (-0.037). The $\mathrm{R}$ value of the final model resulted as 0.6 and the predictable performance of the employees is 37 per cent $(\mathrm{R} 2=0.37)$ from the selected variables with significant at 0.05 per cent level $(\mathrm{p}=0.046)$. The model proves that majority of the training cost spent on the employees can be reduced by the firms with enhancement of productivity.

Table 1. The association between the recruitment and performance variables of the employees.

\begin{tabular}{|c|c|c|c|c|c|}
\hline Performance & Recruitment & $\begin{array}{l}\text { Sum of } \\
\text { Squares }\end{array}$ & $\begin{array}{c}\text { Mean } \\
\text { Square }\end{array}$ & $\mathbf{F}$ & Sig. \\
\hline \multirow{3}{*}{ Reliability } & Between Groups & 201.795 & 67.265 & \multirow{3}{*}{12.426} & \multirow{3}{*}{$(0.000)^{\star \star}$} \\
\hline & Within Groups & 2360.159 & 5.413 & & \\
\hline & Total & 2561.955 & & & \\
\hline \multirow{3}{*}{ Productivity } & Between Groups & 550.308 & 183.436 & \multirow{3}{*}{43.625} & \multirow{3}{*}{$(0.000)^{\star \star}$} \\
\hline & Within Groups & 1833.308 & 4.205 & & \\
\hline & Total & 2383.616 & & & \\
\hline \multirow{3}{*}{ Automation } & Between Groups & 736.506 & 245.502 & \multirow{3}{*}{28.695} & \multirow{3}{*}{$(0.000)^{\star *}$} \\
\hline & Within Groups & 3730.291 & 8.556 & & \\
\hline & Total & 4466.798 & & & \\
\hline \multirow{3}{*}{ Training } & Between Groups & 457.321 & 152.440 & \multirow{3}{*}{36.798} & \multirow{3}{*}{$(0.000)^{\star *}$} \\
\hline & Within Groups & 1806.204 & 4.143 & & \\
\hline & Total & 2263.525 & & & \\
\hline \multirow{3}{*}{ Gamification } & Between Groups & 80.124 & 26.708 & \multirow{3}{*}{7.097} & \multirow{3}{*}{$(0.000)^{\star \star}$} \\
\hline & Within Groups & 1640.765 & 3.763 & & \\
\hline & Total & 1720.889 & & & \\
\hline
\end{tabular}

**Significant at 0.001 per cent level. Source: Computed based on primary data. 
Table 2. Multiple linear stepwise regression analysis of variables to predict the performance.

\begin{tabular}{|c|c|c|c|c|c|c|c|c|c|c|}
\hline Model & VARIABLES & Productivity & Training & Automation & Reliability & Gamification & $R$ & $R 2$ & F Change & Sig. \\
\hline & $B$ & 0.13 & & & & & & & & \\
\hline \multirow[t]{3}{*}{1} & $S E B$ & 0.013 & & & & & 0.44 & 0.2 & 107.186 & $(0.000)^{\star *}$ \\
\hline & $\beta$ & $0.443^{* \star}$ & & & & & & & & \\
\hline & $B$ & 0.117 & 0.091 & & & & & & & \\
\hline \multirow[t]{3}{*}{2} & $S E B$ & 0.012 & 0.012 & & & & 0.54 & 0.29 & 55.752 & $(0.000)^{\star *}$ \\
\hline & $\beta$ & $0.399^{\star *}$ & $0.305^{\star *}$ & & & & & & & \\
\hline & $B$ & 0.081 & 0.124 & -0.062 & & & & & & \\
\hline \multirow[t]{3}{*}{3} & $S E B$ & 0.013 & 0.013 & 0.01 & & & 0.59 & 0.35 & 41.655 & $(0.000)^{\star *}$ \\
\hline & $\beta$ & $0.275^{\star *}$ & $0.414^{* *}$ & $-0.292^{\star *}$ & & & & & & \\
\hline & $B$ & 0.078 & 0.105 & -0.069 & 0.036 & & & & & \\
\hline \multirow[t]{3}{*}{4} & $S E B$ & 0.013 & 0.015 & 0.01 & 0.014 & & 0.6 & 0.36 & 6.494 & $(0.011)^{\star *}$ \\
\hline & $\beta$ & $0.268^{* *}$ & $0.349^{* *}$ & $-0.323^{* *}$ & 0.127 & & & & & \\
\hline & $B$ & 0.084 & 0.116 & -0.065 & 0.045 & -0.037 & & & & \\
\hline \multirow[t]{2}{*}{5} & $S E B$ & 0.013 & 0.016 & 0.01 & 0.015 & 0.018 & 0.6 & 0.37 & 3.998 & 0.046 \\
\hline & $\beta$ & $0.287^{* *}$ & $0.388^{* *}$ & $-0.306^{\star *}$ & 0.159 & -0.107 & & & & \\
\hline
\end{tabular}

**Significant at 0.001 per cent level. Source: Computed based on primary data.

\section{Results}

One-way ANOVA was conducted to compare the effect of Reliability, Productivity, Automation, Training and Gamification when the artificial intelligence is adopted, combined and traditional methods are followed in recruitment. An analysis of variance showed that the effect of reliability on recruitment was significant, $(\mathrm{F}(3,436)=12.426, \mathrm{p}=0.000)$, productivity was significant, $(\mathrm{F}(3,436)=$ 43.625, $\mathrm{p}=0.000)$, automation was significant, $(\mathrm{F}(3,436)=28.695, \mathrm{p}=0.000)$, training was significant, $(\mathrm{F}(3,436)=36.798, \mathrm{p}=0.000)$, and gamification was significant $(\mathrm{F}(3,436)=7.097, \mathrm{p}=0.000)$.

A multiple linear regression equation was calculated to predict the performance of the employees based on indicating variables. The following is the equation:

$$
Y=1.505+0.084 X_{1}+0.116 X_{2}-0.065 X_{3}+0.045 X_{4}-0.037 X_{5} .
$$

$Y$ represents the performance of the employees. 1.505 is the constant value consisting of " $X_{1}$ ” being Productivity, " $X_{2}$ ” being Training, " $X_{3}$ ” being Automation, " $X_{4}$ ” being Reliability and " $X_{5}$ ” being Gamification. A significant regression equation was found $\left(F(5,434)=49.905\right.$, $(\mathrm{p}<0.000)$, with an $\mathrm{R}^{2}$ of 0.365 . Hence predicted performance is equal to $1.505+0.084$ (Productivity) +0.116 (Training) -0.065 (Automation) +0.045 (reliability) -0.037 (Gamification). The performance of the employees increased for every 0.084 hours of the productivity in, reliability in quality activities, training in 0.116 number of programmes, reduced the 0.065 cost of operations by automation, and 0.037 cost by gamification. The error terms are cost of implementation, adaptability of employees, time 
and resources involved in training the employees to adopt this technology. Hence productivity, reliability, automation, and Training were significant predictors of performance among the employees.

\section{Findings \& Discussions}

The performance of the employees increased per unit for every 0.130 hours of the productivity in model 1 . The performance of the employees increased per unit for every 0.117 hours of the productivity along with 0.091 training programmes as per model 2. The performance of the employees increased per unit for every 0.081 hours of the productivity, 0.124 training programmes and decreased workload by 0.062 when automated as per model 3 . As per model 4 , the performance of the employees increased per unit for every 0.078 hours of the productivity, 0.105 of training programmes, decreased workload by 0.069 when automated and reliability on the reports generated based on the integration of technology increases by 0.036 . The performance of the employees increased for every 0.084 hours of the productivity in, reliability in quality, levels of training programmes by 0.116 , reduced the 0.065 workloads in the operations by automation, and 0.037 by the gamification. However, there is a partial significant effect of adapting AI in gamification on performance as the coefficient $(0.046<$ 0.05). A One-way ANOVA was conducted to compare the effect of performance indicating variables with recruitment based on the adaptability of artificial intelligence resulting to be a greater association. Hence null hypothesis has been rejected.

\section{Conclusion}

Artificial intelligence has been the most apt innovative technology that facilitates in better recruitment process when implemented strategically having a significant effect on the performance of the employees. Increase in productivity, training programmes, automation of the process and the reliability of reports improves the performance of employees. Hence the adaptability of this technology results in better performance of the employees and performance indicating variables are highly significant predictors.

\section{Conflicts of Interest}

The authors declare no conflicts of interest regarding the publication of this paper.

\section{References}

[1] Melder, B. (2018) The Role of Artificial Intelligence (AI) in Recruitment. https://www.talentlyft.com/en/blog/article/207/the-role-of-artificial-intelligence-ai-i $\underline{\text { n-recruitment }}$

[2] Goyal, M. (2017) How Artificial Intelligence Is Reshaping Recruitment, and What It Means for the Future of Jobs.

https://economictimes.indiatimes.com/jobs/how-artificial-intelligence-is-reshapingrecruitment-and-what-it-means-for-the-future-of-jobs/articleshow/60985946.cms 
[3] Rafter, R., Bradley, K. and Smyth, B. (2000) Automated Collaborative Filtering Applications for Online Recruitment Services. In International Conference on Adaptive Hypermedia and Adaptive Web-Based Systems, Springer, Berlin, Heidelberg, 363-368. https://doi.org/10.1007/3-540-44595-1_48

[4] Khosla, R. (2003) An Online Multi-Agent E-Sales Recruitment System. Proceedings of 2003 IEEE/ WIC International Conference on Web Intelligence (WI 2003), Halifax, NS, 13-17 October 2003, 111-117. https://doi.org/10.1109/WI.2003.1241181

[5] Färber, F., Weitzel, T. and Keim, T. (2003) An Automated Recommendation Approach to Selection in Personnel Recruitment. AMCIS 2003 Proceedings, North America, 31st December 2003, 302.

[6] Dreyfus-León, M. and Chen, D.G. (2007) Recruitment Prediction with Genetic Algorithms with Application to the Pacific Herring Fishery. Ecological Modelling, 203, 141-146. https://doi.org/10.1016/j.ecolmodel.2005.09.016

[7] Loreto, V. and Steels, L. (2007) Social Dynamics: Emergence of Language. Nature Physics, 3, 758-760. https://doi.org/10.1038/nphys770

[8] Faliagka, E., Iliadis, L., Karydis, I., Rigou, M., Sioutas, S., Tsakalidis, A. and Tzimas, G. (2014) On-Line Consistent Ranking on E-Recruitment: Seeking the Truth behind a Well-Formed CV. Artificial Intelligence Review, 42, 515-528.

https://doi.org/10.1007/s10462-013-9414-y

[9] Russell, S.J. and Norvig, P. (2016) Artificial Intelligence: A Modern Approach. Pearson Education Limited, Malaysia. 